\title{
Specimen Referral Network to Rapidly Scale-Up CD4 Testing: The Hub and Spoke Model for Haiti
}

Frantz Jean Louis ${ }^{1 *}$, Anna Janick Osborne ${ }^{2}$, Viala Jean Elias ${ }^{3}$, Josiane Buteau ${ }^{3}$, Jacques Boncy ${ }^{3}$, Angela Elong ${ }^{4}$, Amber Dismer ${ }^{5}$, Vikram Sasi $^{3}$, Jean Wysler Domercant ${ }^{1}$, Daniel Lauture ${ }^{6}, \mathbf{S}$ Arunmozhi Balajee ${ }^{5}$ and Barbara J Marston ${ }^{5}$

${ }^{1}$ Centers for Diseases Control and Prevention, Port-au-Prince, Haiti

${ }^{2}$ Clinton Health Access Initiative, Port-au-Prince, Haiti

${ }^{3}$ Laboratoire National de Santé Publique, Ministry of Health, Government of Haiti, Port-au-Prince, Haiti

${ }^{4}$ Partnership for Supply Chain Management, Port-au-Prince, Haiti

${ }^{5}$ Centers for Diseases Control and Prevention, Atlanta, Georgia, USA

${ }^{6}$ Unite de Gestion des Programmes, Ministry of Health, Government of Haiti, Port-au-Prince, Haiti

\begin{abstract}
Objectives: Regular and quality CD4 testing is essential to monitor disease progression in people living with HIV. In Haiti, most laboratories have limited infrastructure and financial resources and have relied on manual laboratory techniques. We report the successful implementation of a national specimen referral network to rapidly increase patient coverage with quality CD4 testing while at the same time building infrastructure for referral of additional sample types over time.
\end{abstract}

Method: Following a thorough baseline analysis of facilities, expected workload, patient volumes, cost of technology and infrastructure constraints at health institutions providing care to HIV patients, the Haitian National Public Health Laboratory designed and implemented a national specimen referral network. The specimen referral network was scaled up in a step-wise manner from July 2011 to July 2014.

Results: Fourteen hubs serving a total of 67 healthcare facilities have been launched; in addition, 10 healthcare facilities operate FACSCount machines, 21 laboratories operate PIMA machines, and 11 healthcare facilities are still using manual CD4 tests. The number of health institutions able to access automated CD4 testing has increased from 27 to $113(315 \%)$. Testing volume increased $76 \%$ on average. The number of patients enrolled on ART at the first healthcare facilities to join the network increased $182 \%$ within 6 months following linkage to the network. Performance on external quality assessment was acceptable at all 14 hubs.

Conclusion: A specimen referral network has enabled rapid uptake of quality CD4 testing, and served as a backbone to allow for other future tests to be scaled-up in a similar way.

Keywords: Specimen referral network; CD4 testing; Hubs; Spokes; External quality assessment

\section{Introduction}

Unprecedented efforts and investment have led to drastic scale up of care and treatment for people living with HIV/AIDS in resource-limited countries (RLC) over the last decade. In 2013, the Joint United Nations Program on HIV/AIDS (UNAIDS) reported More than 9.7 million people as of 2013, in low- and middle-income countries are receiving lifelong antiretroviral therapy (ART) resulting in significant decline in morbidity and mortality [1]. Haiti is affected by the most pervasive HIV epidemic outside of sub-Saharan Africa, but a range of prevention and treatment efforts have been implemented, and the HIV prevalence in the general population has been stable over the past five years [2]. As of late 2013, more than 50,000 HIV-infected persons in Haiti were receiving ART, meeting more than $70 \%$ of the estimated need based on most recent national guidelines [1] The World Health Organization (WHO) guidelines recommend a minimum package of laboratory tests for patients with HIV that includes CD4 cell count testing [3]. Access to measurements of $\mathrm{CD} 4$ cell counts is vital for determining the level of immune suppression and predicting host susceptibility to specific opportunistic infections [4-6]. Where resources are insufficient to ensure universal treatment access, the results of CD4 testing are also used to prioritize patients for treatment, thus CD4 testing is a gateway to early ART, which in turn reduces morbidity and mortality, decreases mother-to-child transmission of HIV and decreases the incidence of HIV-associated tuberculosis (TB) [7,8]. CD4 counts are also used as a tool to monitor disease progression and the effectiveness of ART, particularly in Haiti and other countries where viral load testing is not widely available [9]. However, there are important challenges limiting widespread access to CD4 cell count testing, including inconsistent supplies of electricity and water, poor physical infrastructures, shortage of skilled technicians, an inconsistent supply-chain for consumables and reagents, and inadequate equipment maintenance.

The need to increase availability of CD4 testing in RLC has driven the development of an array of cost-efficient and technically simple CD4 cell count testing platforms [4]. Two possible approaches to expansion of access to CD4 tests in RLC include establishment of decentralized testing using these platforms and development of testing networks that support transport of samples from peripheral healthcare facilities to centralized testing laboratories [10].

During initial expansion of HIV care and treatment services in Haiti, most laboratories relied on a manual method of CD4 testing based

*Corresponding author: Frantz Jean Louis, Centers for Diseases Control and Prevention, Port-au-Prince, Haiti, Tel : (509) 31703488; E-mail: yjeanlouis@cdc.gov

Received May 19, 2015; Accepted June 16, 2015; Published June 26, 2015

Citation: Jean Louis F, Osborne AJ, Elias VJ, Buteau J, Boncy J, et al. (2015) Specimen Referral Network to Rapidly Scale-Up CD4 Testing: The Hub and Spoke Model for Haiti. J AIDS Clin Res 6: 488. doi:10.4172/2155-6113.1000488

Copyright: (c) 2015 Jean Louis F, et al. This is an open-access article distributed under the terms of the Creative Commons Attribution License, which permits unrestricted use, distribution, and reproduction in any medium, provided the original author and source are credited. 
on microscopic evaluation of beads coated with antibodies directed against the $\mathrm{CD} 4$ and $\mathrm{CD} 8$ receptors on $\mathrm{T}$ lymphocytes (cytosphere assay; Coulter Corporation, Hialeah, Fla.). While the manual CD4 requires minimal equipment beyond a low-cost microscope and can be performed at remote health care facilities, the method has low throughput (approximately 10 samples per day), is labor intensive and the quality of the result is hard to ensure due to the subjectivity introduced when cells are counted visually [11]. By 2010 , only $12 \%$ of the CD4 testing needs in Haiti were being met with automated equipment and very few laboratories had infrastructure that would support automation of laboratory testing.

Many of the general challenges to expanding access to CD4 testing in RLC are specifically relevant in Haiti. Most laboratories in the country depend on the national electric grid which is not constant. In Haiti, blackouts and brownouts are common, the national utility provider is not able to meet the national demand for electricity, road conditions are poor and some facilities can only be reached by motorcycle. However, Haiti has a well-functioning, centralized system for drugs and laboratory commodities management for HIV care and treatment programs and a national public health laboratory (French acronym: LNSP) with good capacity for testing and expanding capacity related to implementation of national level laboratory programs.

In 2011, in order to expand access to quality CD4 cell count testing, Haiti established a specimen referral network (SRN) based mainly on a model of established central testing "hubs" serving more peripheral "spoke" laboratories/healthcare facilities. Needs not met through specimen referral were addressed using automated point-of-care testing equipment. While the SRN is meant to support transport of multiple types of laboratory samples over time, the primary initial objective was to support $80 \%$ of CD4 testing needs in the country. We describe of the establishment of the CD4 testing system and progress in expanding access to CD4 testing in Haiti.

\section{Methods}

\section{Establishment of the CD4 cell count testing network}

The first step in the establishment of the network was to create a logistics and coordination unit (LCU) at LNSP in early 2011. This unit was responsible for selecting the participating laboratories based on infrastructure, human resources, and location; for the unit was also charged with training laboratory technicians and specimen transporters, and for monitoring daily activity of the network.

In the $2^{\text {nd }}$ quarter of 2011, the LCU, in collaboration with staff from the Clinton Health Access Initiative (CHAI) and American Public Health Laboratory (APHL), conducted an assessment of 20 laboratories to determine their potential to serve as testing hubs. A team assessed infrastructure, human resources, and laboratory capacity using the WHO/African Regional (AFRO) Laboratory Quality Improvement Process Towards Accreditation (SLIPTA) check list [12]. Following this assessment, 14 laboratories were chosen (Figure 1) as testing hubs based on whether they had or could reasonably be upgraded to have adequate space, constant electricity, running water, functioning air conditioning, available cold chain storage, and trained technicians. Once hub laboratories had been identified, referrals, or "spoke" facilities, were chosen with a travel time of less than 60 minutes from the corresponding hub. This generated 14 "circuits", each composed of a hub laboratory with automated CD4 testing capacity (a laboratory with a FACSCount machine) and 4-8 spokes. Laboratories that could not be reasonably linked to hub laboratories because of transport challenges were chosen to receive point of care equipment in an effort to allow the broadest possible access to $\mathrm{CD} 4$ cell count testing.

The next step was to ensure that identified hub laboratories had adequate infrastructure to support automated CD4 cell count testing. Electrical systems at hub laboratories were upgraded as needed with installation of back-up power sources, surge protection equipment, and inverters designed to safely power down equipment during interruptions in the main power supply. Air conditioning was installed at 4 hubs. Anticipated workloads were estimated based on the patient volume from the spoke laboratories and the pre-network testing volume at hub healthcare facilities; additional technicians were recruited in line with these estimates.

Twenty-four FACSCount (Becton Dickinson, San Jose, CA) machines and 21 PIMA (Alere Inc. Waltham, MA.) machines were installed. To ensure maximum performance of the equipment, service contracts were established with in-country representatives of Alere and BD Dickenson for preventive maintenance and repair of all FACSCount and PIMA in the network. Initial supplies needs were quantified based on anticipated testing volumes. Twenty five laboratory technicians were trained on automated CD4 testing using FACSCount and 21 laboratories received onsite training for the use of the PIMA pointof-care machines. Twenty-eight drivers were trained in safe specimen packing and handling practices, transport and decontamination procedures in case of spillage. Healthcare facilities were provided with packaging materials for sample transport. Detailed standard operating procedures (SOPs) for specimen shipping, reception, rejection and for incident reports were developed.

Accurate CD4 T-cell enumeration is pivotal for correct clinical management of HIV/AIDS patients. An external quality assurance (EQA) program is critical to ensure that patients are accurately monitored on therapy, irrespective of methodology or geographic location [13]. To assess the quality of laboratory performing CD4 test, all hub laboratories were enrolled in Haiti's EQA program (biannual testing of standard panels provided by Quality Assessment and Standardization for Immunological measures relevant to HIV (QASI) [14] as they were linked to the SRN. Laboratories with point-of-care CD4 were also enrolled in Haiti EQA program.

The SRN was piloted in July 2011 with LNSP as a testing hub and 4 nearby laboratories delivering specimens daily for testing at LNSP and expanded nationally.

\section{Evaluation of the impact of network establishment}

Information about the networked healthcare facilities and other routine monitoring data were analyzed to evaluate the impact of the SRN. Changes in national access to CD4 testing were analyzed for the period from December 2008 to August 2013. Testing volumes were analyzed for 10 circuits that had 6 months of follow up data by the time of the evaluation; data on testing volume 6 months prior to and 6 months after establishment of these circuits were analyzed. Information about the number of patients with $2 \mathrm{CD} 4$ tests a year and the numbers of patients enrolled on ART was extracted from national program reports for 9 healthcare facilities that had a full year of post-network data on CD4 testing at the time of the evaluation. The impact of CD4 testing automation on the quality of laboratory results was assessed based on analysis of results of EQA evaluations; trimmed mean and standard deviations for all participating health care facilities around the world were provided by QASI for low and normal proficiency tests and LevyJennings charts were generated to evaluate the performance of all 14 hubs for the last QASI session in 2013. Data were analyzed using SPSS 


\section{CD4 Specimen Referral Network in Haiti}

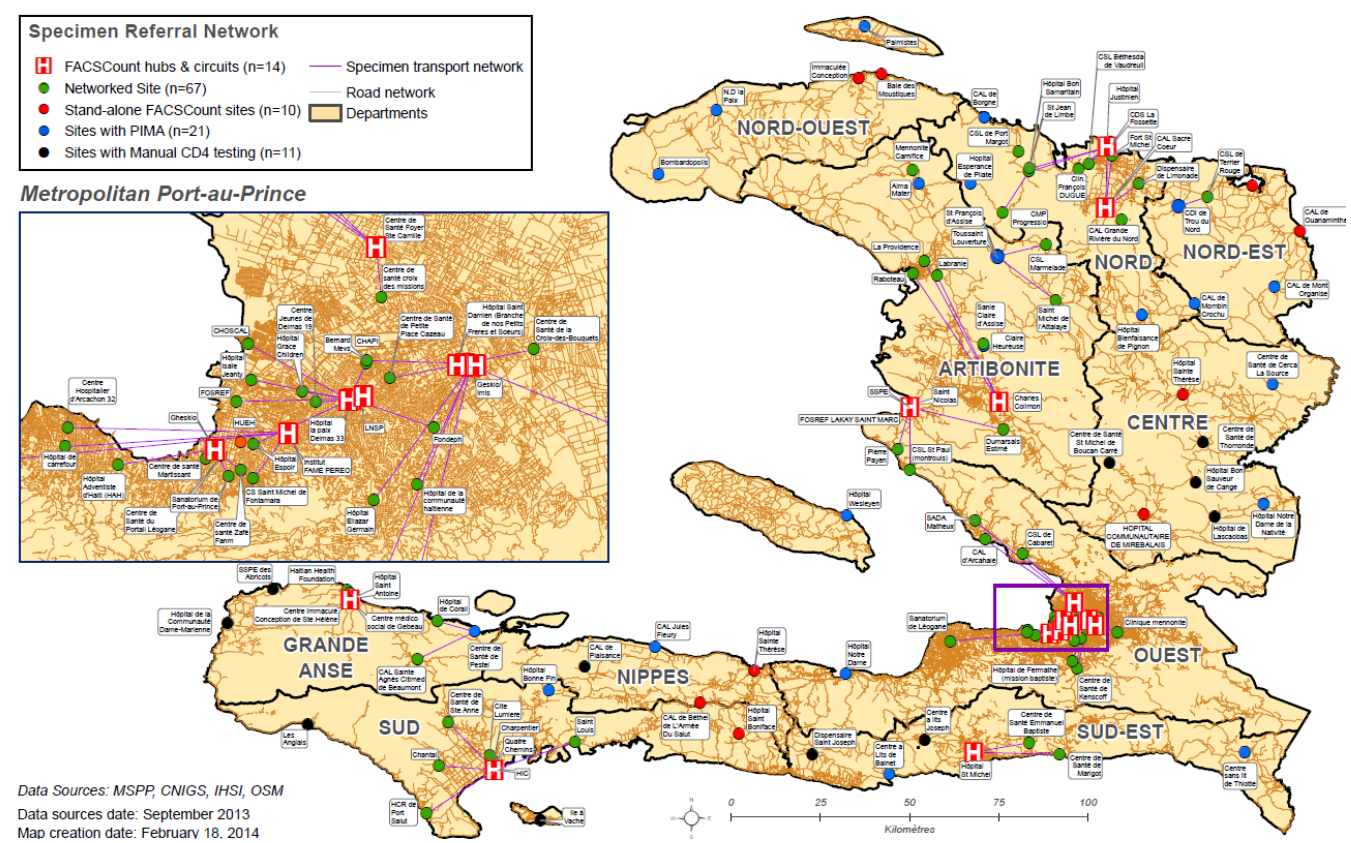

Figure 1: The Haitian specimen referral network map. Location for the14 FACSCount Hubs, the 67 spoke laboratories, the 31 healthcare facilities using PIMA or FACSCount and the 11 facilities still using manual CD4 are indicated as shown in the legend.

(SPSS, v13.0 Chicago: SPSS Inc.) and ArcGIS (Esri, Redlands, CA), p values of $<.05$ (Student $t$ test) were considered significant.

\section{Results}

Three years after the launch of the pilot, all 14 planed circuits were operational, serving a total of 67 healthcare facilities. These circuits are shown in Figure 1. A total of 24 laboratories have FACSCount CD4 equipment [14] hubs and 10 additional standalone FACSCount), 21 laboratories are operating PIMA machines and 11 are still using manual CD4 tests. Of 123 healthcare facilities providing care and treatment for HIV infected patients in Haiti, 112 (91\%) have access to automated CD4 testing as of December 2013 (14 hubs, 67 spokes, 10 labs with standalone FACSCount and 21 one labs with PIMA). Access to automated CD4 testing was $31 \%$ (14 of 45 facilities) in December 2008, 30\% (14 of 46) in December 2009, 29\% (15 of 51) in December 2010, 40\% (27 of 61) in December 2011, and 52\% (44 of 84) in December 2012 (Figure 2). The 112 healthcare facilities covered by automated CD4 testing provide care to approximately $99 \%$ (55606/56205) of the total of HIV infected patients enrolled and receiving ART in Haiti.

A significant increase $(76 \%, p=0.007$, Student $t$ test $)$ in the total volume of CD4 tests was observed comparing data 6 months before (6972 CD4 tests from 68 peripheral health care facilities) and 6 months after (12296 CD4 tests) healthcare facilities joined the SRN. Testing volumes for the first ten established circuits are shown in Figure 3; for two circuits, no testing was conducted prior establishment of the network. At the remaining healthcare facilities, the increase in CD4 total testing volume ranged between and 35\% (circuit 4) and 239\% (circuit 3).

The percentage of patients with 2 CD4 tests per year at the first 9 healthcare centers (spokes) enrolled in the SRN is shown in Table 1. For 8 of the 9 healthcare centers, the absolute change in percentages was a median of $21 \%$. Only one facility had a drop in coverage. The overall median change was $+19 \%$ (Table1). The number of patients enrolled on ART during the same time period increased from 8,318 to 23, 446 (182\% increase).

All 14 testing hubs have been enrolled in the biannual EQA program. Analysis of results from the CD4 EQA program from QASI database showed that values for low and normal CD4 proficiency test fall within the recommended \pm 2 SD from the mean for all health care facilities (Figure 4).

\section{Discussion}

We report the successful implementation of a national specimen referral network with decentralized access to laboratory testing as suggested by Nkengsong [15]. We observed rapid expansion in CD4 testing with the establishment of the SRN in Haiti, from $40 \%$ of ART sites in 2010 having access to automated CD4 testing, to $91 \%$ in 2013. We further observed a marked increase in the number of new patients enrolled for ART care and treatment one year after implementation of the network. The SRN in Haiti has not only had an important impact on CD4 test availability countrywide, but it has allowed stakeholders to focus efforts to improve laboratory infrastructure. All 14 testing hubs have benefited from this particular attention and there have been improvements in electrical systems, functional air conditioning, running water and adequate space for laboratory testing. While we are unable to specifically compare the accuracy of testing prior to and following the establishment of the network, current EQA results reflect high-quality testing.

The choice to establish an SRN offered an opportunity to standardize laboratory equipment across the country, streamline management of the supply chain, and increase communication between laboratories in the network and at the same time to ensure good quality of results 


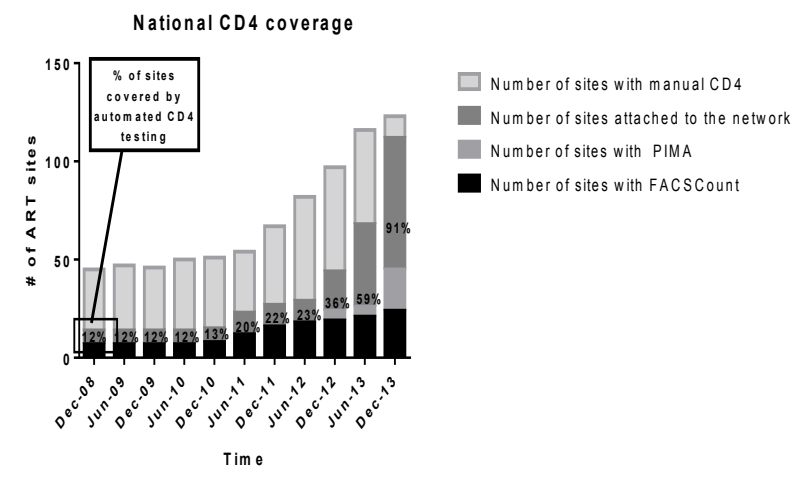

Figure 2: National CD4 coverage. Comparison of the number of health care facilities with CD4 testing capacity (manual vs automated) in Haiti from 2008 to 2013

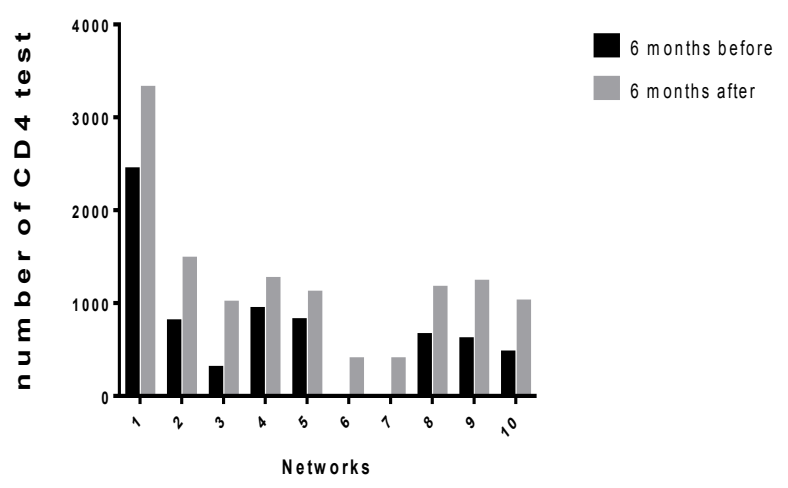

Figure 3: Impact of the specimen referral network on CD4 testing volume. We compared the number of CD4 tests performed by the first 10 networks (average of 5.5 healthcare facilities per network) 6 months prior to and 6 months after integrating the specimen referral network.

\begin{tabular}{|c|c|c|c|c|c|}
\hline $\begin{array}{l}\text { Healthcare } \\
\text { center }\end{array}$ & Year 1 & Year 2 & $\begin{array}{l}\text { Absolute } \\
\text { Change }\end{array}$ & $\begin{array}{l}\text { Patients } \\
\text { on } \mathrm{ART}^{2} \text { at } \\
\text { Year1 }\end{array}$ & $\begin{array}{c}\text { Patients on ART at } \\
\text { Year2 }\end{array}$ \\
\hline 1 & 9.2 & 51.9 & 42.7 & 2875 & 3052 \\
\hline 2 & 36.2 & 54 & 17.8 & 1009 & 1024 \\
\hline 3 & 7 & 35.9 & 28.9 & 1084 & 1332 \\
\hline 4 & 57.1 & 26.7 & -30.4 & 60 & 114 \\
\hline 5 & 46.8 & 71.4 & 24.6 & 691 & 694 \\
\hline 6 & 7.6 & 21.8 & 14.2 & 1335 & 15540 \\
\hline 7 & 22.3 & 36.7 & 14.4 & 522 & 569 \\
\hline 8 & 25.4 & 65.3 & 39.9 & 686 & 865 \\
\hline 9 & 1 & 12.8 & 11.8 & 56 & 256 \\
\hline \multicolumn{3}{|c|}{ Positive only median } & 21.2 & & \\
\hline \multicolumn{3}{|c|}{ Total median } & 17.8 & & \\
\hline \multicolumn{4}{|c|}{ Total } & 8318 & 23446 \\
\hline \multicolumn{6}{|c|}{$\begin{array}{l}\text { 1SRN: Specimen Referral Network } \\
{ }^{2} \text { ART: Antiretroviral therapy }\end{array}$} \\
\hline
\end{tabular}

Table 1. Impact of the SRN on CD4 testing and patients enrollment on ART. Comparison of the percentage of patients with 2 CD4 tests a year, one year prior to (Year1) and one year after (Year2) establishment of the SRN ${ }^{1}$ from the first 9 health care facilities enrolled in the SRN.

by enrolling all the hubs in an external quality assessment program. While we have not done a formal cost analysis, we believe that the implementation of the SRN was a cost effective way to ensure access to not only CD4 cell count testing but to also serve as a backbone

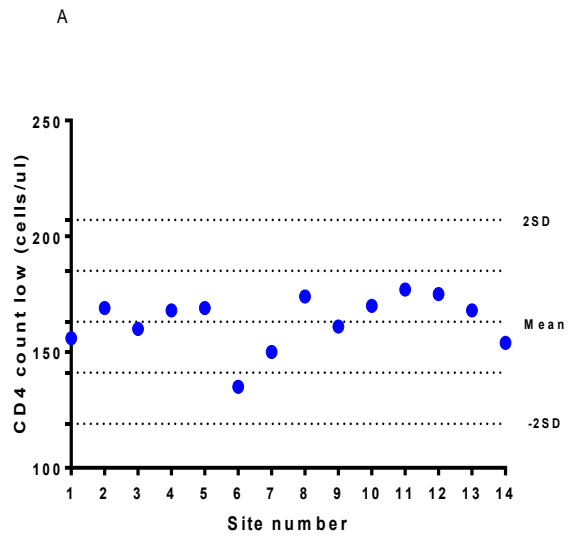

B

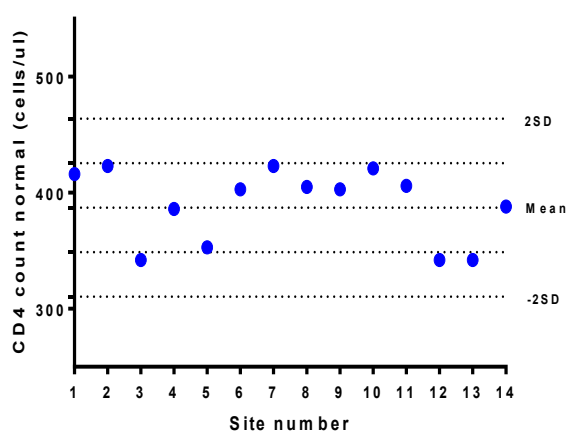

Figure 4: Levy-Jennings plot demonstrating CD4+T-lymphocytes absolute counts (two level control: for low (A) and normal (B) lymphocyte absolute counts) obtained from Trial 34 on October 2013. Each plot shows the concurrent results of CD4 EQA from the QASI program for all 14 Hubs.

for HIV viral load testing and MDR TB surveillance; with increased transportation costs being offset by lower equipment, maintenance, and human resource costs than would have been incurred with broader distribution of equipment.

Other countries have used different strategies to decentralize access to $\mathrm{CD} 4$ testing. Capacity for CD4 testing was strengthened in 6 African countries through distribution of flow cytometers at 122 laboratories [6]. The use of a SRN in Haiti (as compared to distribution of machines to large numbers of health care facilities) may facilitate QA/QC and service and maintenance of equipment, supplies distribution, training and staff retention since only 14 testing hubs support more than $90 \%$ of the national CD4 testing needs.

Several African countries are implementing SRNs. In Zambia, a SRN has been designed to support enrollment in care and monitoring of HIV+ pregnant women; in Nyanza, a rural province of Kenya, a 35\% upsurge in the number of patients enrolled on ART was observed only 5 months after implementing a SRN; more recently Uganda reported a successful implementation of a specimen transport system which increased access to early infant diagnosis of HIV ranging from $36 \%$ to $51 \%[16-18]$.

An important advantage of the SRN is that it can be adapted for use for other types of testing. The SRN in Haiti has grown not only in terms 
of number of laboratories covered, but also in terms of the number of different type of tests being offered to the population. In July 2013, LNSP introduced HIV viral load testing for better monitoring of HIV infected patients; the SRN has allowed 5 high patient-volume hospitals to benefit from viral load testing. The SRN also offers a platform for integrated laboratory-based surveillance. There are plans to further link "hub" facilities to the regional and central reference laboratories, and further expand the types of samples transported (for example to include transport of sputum samples for testing for drug-resistant TB, and slides for malaria and TB slide rechecking for external quality assurance programs).

The sustainability of the SRN remains a big challenge. The SRN is supported with external funding with little financial involvement of the Haitian ministry of health. Efforts are ongoing to decrease the cost associated with the SRN with more motorcycles in the circuits instead of cars (where possible) and more involvement of local stakeholders at the different healthcare facilities being served by the SRN. The Haiti model is among the first reports to describe the influence of an SRN on testing volumes. Our analysis is limited because as an observational evaluation, we cannot rule out the influence of other factors on testing frequency or enrolment of patients on ART. 5.

\section{Conclusion}

The SRN has enabled rapid uptake of quality CD4 testing in Haiti through a combination of specimen transport from lower level peripheral health care facilities to well-equipped testing hubs and point of care testing at health care facilities with difficult road access. The experience in Haiti serves as a practical example of establishing an SRN in a resource limited setting.

\section{References}

1. The Joint United Nations Program on HIVIAIDS (UNAIDS) (2013) UNAIDS report on the global AIDS epidemic.

2. Ministeredela Sante Publique et de la Population (2013) Enquête Mortalité, Morbidité et Utilisation des Services EMMUS-V, Haiti 2012.

3. World Health Organization (2013) Antiretroviral therapy for HIV infection in adults and adolescents.

4. Belec L, Bonn JP (2011) Challenges in implementing HIV laboratory monitoring in resource-constrained settings how to do more with less. Future Microbiol6: 1251-1260.

5. Carter RJ, Dugan K, El-Sadr WM, Myer L, Otieno J et al. (2010) CD4(+) Cell Count Testing More Effective Than HIV Disease Clinical Staging in Identifying
Pregnant and Postpartum Women Eligible for Antiretroviral Therapy in Resource-Limited Settings. Jaids-Journal of Acquired Immune Deficiency Syndromes55: 404-410.

6. Marinucci F, Medina-Moreno S, Paterniti AD, Wattleworth M, Redfield RR 2011) Decentralization of CD4 testing in resource-limited settings 7 years of experience in six African countries. Cytometry A79: 368-374.

7. Zachariah R, Reid SD, Chaillet P, Massaquoi M, Schouten EJ et al. (2011) ViewpointWhy do we need a point-of-care CD4 test for low-income countries? Tropical Medicine \& International Health16: 37-41.

8. Eholie SP, Aoussi FE, Ouattara IS, Bissagnene E, Anglaret X (2012) HIV treatment and care in resource-constrained environments challenges for the next decade. Journal of the International AIDS Society 15: 17334.

9. Gilks CF, Crowley S, Ekpini R, Gove S, Perriens J, Souteyrand Y, et al. (2006) The WHO public-health approach to antiretroviral treatment against HIV in resource-limited settings. Lancet 368: 505-510.

10. Gerlach JB, David, Domingo, Gonzalo, Weigl, et al. (2008) Increased access to diagnostic tests for HIV case management. In: aids 2031 Science and Technology Working Group.

11. Vajpayee M, Mohan (2011) T Current practices in laboratory monitoring of HIV infection. Indian J Med Res134: 801-822.

12. Gershy-Damet GM, Rotz P, Cross D, Belabbes el H, Cham F et al. (2010) The World Health Organization African region laboratory accreditation process improving the quality of laboratory systems in the African region. Am J Clin Pathol 134: 393-400.

13. Glencross DK, Aggett HM, Stevens WS, Mandy F (2008) African regional external quality assessment for CD4 T-cell enumeration: development outcomes and performance of laboratories. Cytometry B Clin Cytom 74 Suppl 1: S69-79.

14. Mandy F, Bergeron M, Houle G, Bradley J, Fahey J (2002) Impact of the international program for Quality Assessment and Standardization for Immunological Measures Relevant to HIVIAIDSQASI. Cytometry50: 111-116.

15. Nkengasong JN (2009) Strengthening laboratory services and systems in resource-poor countries. Am J Clin Pathol 131: 774.

16. Bryan-Mofya G PJ (2011) Increasing access to diagnostics for a mother baby pair through the specimen referral system. 6th IAS Conference on HIV Pathogenesis and Treatment.

17. Opiyo E. NL, Pancras M.E, Omondi J. Marston B, Tukei P (2006) Enhancing access to antiretroviral therapy in Kenya through a specimen referral network for CD4 testing a rural region/province experience. AIDS 2006 - XVI International AIDS Conference.

18. Kiyaga C, Sendagire H, Joseph E, McConnell I, Grosz J, et al. (2013) Uganda's new national laboratory sample transport system a successful model for improving access to diagnostic services for Early Infant HIV Diagnosis and other programs. PLoS One 8: 78609. 